\title{
Anesthesia for patients with PTRF mutations
}

Hirano A. ${ }^{1}$, Takada T. ${ }^{1}$, Busujima Y. ${ }^{2}$, Takahashi H. ${ }^{1}$, Suzuki T. ${ }^{1}$

${ }^{1}$ Tokyo Metropolitan Bokutoh Hospital, Department of Anesthesiology, Tokyo, Japan ${ }^{2}$ Tokyo Metropolitan Bokutoh Hospital, Department of Dentistry and Oral Surgery, Tokyo, Japan

Background
What is PTRF (polymerase I and transcript release factor)?
- PTRF is a caveolar-associated protein.
the stabilization of caveolins. ${ }^{1 \text { ) }}$
What are caveolae?
- Caveolae are invaginations of the plasma membrane.
- They are involved in many cellular processes, including
clathrin-independent endocytosis, cholesterol transport,
and signal transduction. ${ }^{1)}$
What is caveolin?
- Caveolin is a membrane protein that characterizes caveolae. Glia, 2015
- There are 3 subtypes of caveolin: Cav-1, Cav-2, and Cav-3
- Cav-1 and Cav-2 are present in most cell types.
- Cav-1 plays an important role in cholesterol trafficking and homeostasis. ${ }^{2)}$
- Cav-3 is specific to skeletal, smooth, and cardiac muscles.
- Genetic aberrations in Cav-3 are reported to cause muscular disorders. ${ }^{3)}$
- An interaction between the ryanodine receptor and Cav-3 is known.
What are the symptoms of PTRF mutation?
- Congenital generalized lipodystrophy (CGL) developed from infancy.
- Abnormal metabolism of lipids and glucose.
- Muscular disease (muscular hypertrophy, atrophy and mounding).
- Cardiomyopathy, long QT syndrome.
- Renal impairment, IgA nephrosis.
- Immunodeficiency. $\quad$ Few PTRF mutations have been reported,
- Disturbance of growth. $\quad$ but there are no reports regarding anesthesia.

\section{Case report}

A 29-year-old man (height, $126 \mathrm{~cm}$; weight, $22 \mathrm{~kg}$ ) harboring PTRF mutations was scheduled for a mandibular dentigerous cystectomy.

His profile

- Neonatal period: Patient was born with neonatal asphyxia (full term birth, $3720 \mathrm{~g}$ ), had cerebral hemorrhage, and was a 'floppy' infant.

- 4 months of age: Patient underwent muscle biopsy and was diagnosed as having non-Fukuyama dystrophy.

- 3 years of age : Patient began treatment for nephrosis

- 19 years of age: Patient fainted twice. He underwent a Holter ECG exam and was diagnosed as having long QT syndrome (LQTS).

He began to receive medication.

- 22 years of age: Patient was diagnosed, by genetic analysis, with a metabolic disorder related to caveolin owing to PTRF mutations.

- 29 years of age: Patient developed cholecystitis and came to our hospital. The cholecystitis was not clinically urgent, but the purulent cyst needed to be treated.

Primary symptoms

CGL associated with myopathy

Long QT syndrome

Refractory nephrosis

Abnormal lipid metabolism

Skeletal dysplasia

Preoperative examination

Blood exam: TP $6.6 \mathrm{~g} / \mathrm{dl}$, Alb $3.7 \mathrm{~g} / \mathrm{dl}$,

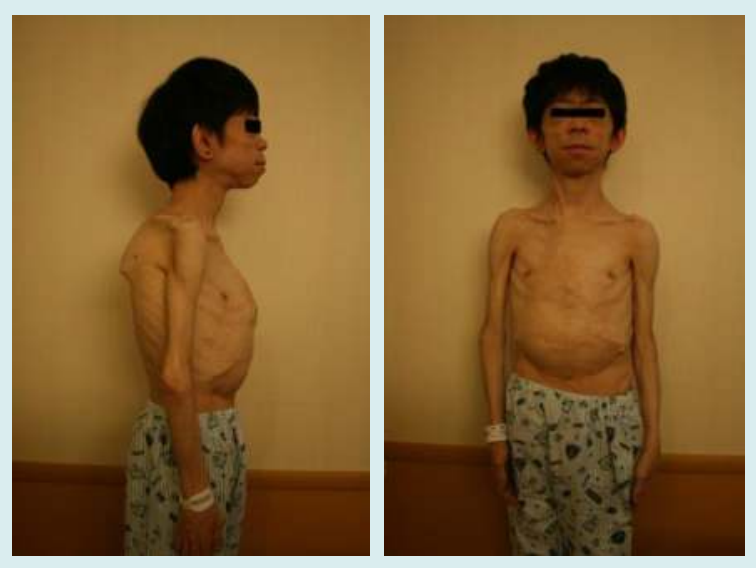

UN 9 mg/dl, Cre 0.2 mg/dl, eGFR 429, CK 282 U/l, T-cho 243 mg/dl

ECG: 66 bpm QT/QTc 370/383z

Medication

methylprednisolone $4 \mathrm{mg}$, mexiletine $100 \mathrm{mg}$, propranolol $10 \mathrm{mg}$,

levocarnitine, alfacalcidol, cyclosporin A, pravastatin

\section{Three important patient concerns}

(1) Avoiding malignant hyperthermia

(2) Concerns about abnormal lipid metabolism

(3) Counterplan for arrhythmia including Torsade de Pointes

Our plan

(1) Avoid exposure to inhalation agents

(2) Avoid using lipid-soluble agents, such as Propofol

(3) Use defibrillation pads and prepare medications for treatment

References

1)Hayashi YK, Matsuda C, Ogawa M, et al. J. Clin. Invest. 2009; 119(9):2623-33.

2)Shi Y, Tan SH, Ng S, et al. Autophagy. 2015,11(5):769-784

3)Song DW, Lee KE, Ryu JY, et al. BBRC. 2015, 463: 975-981
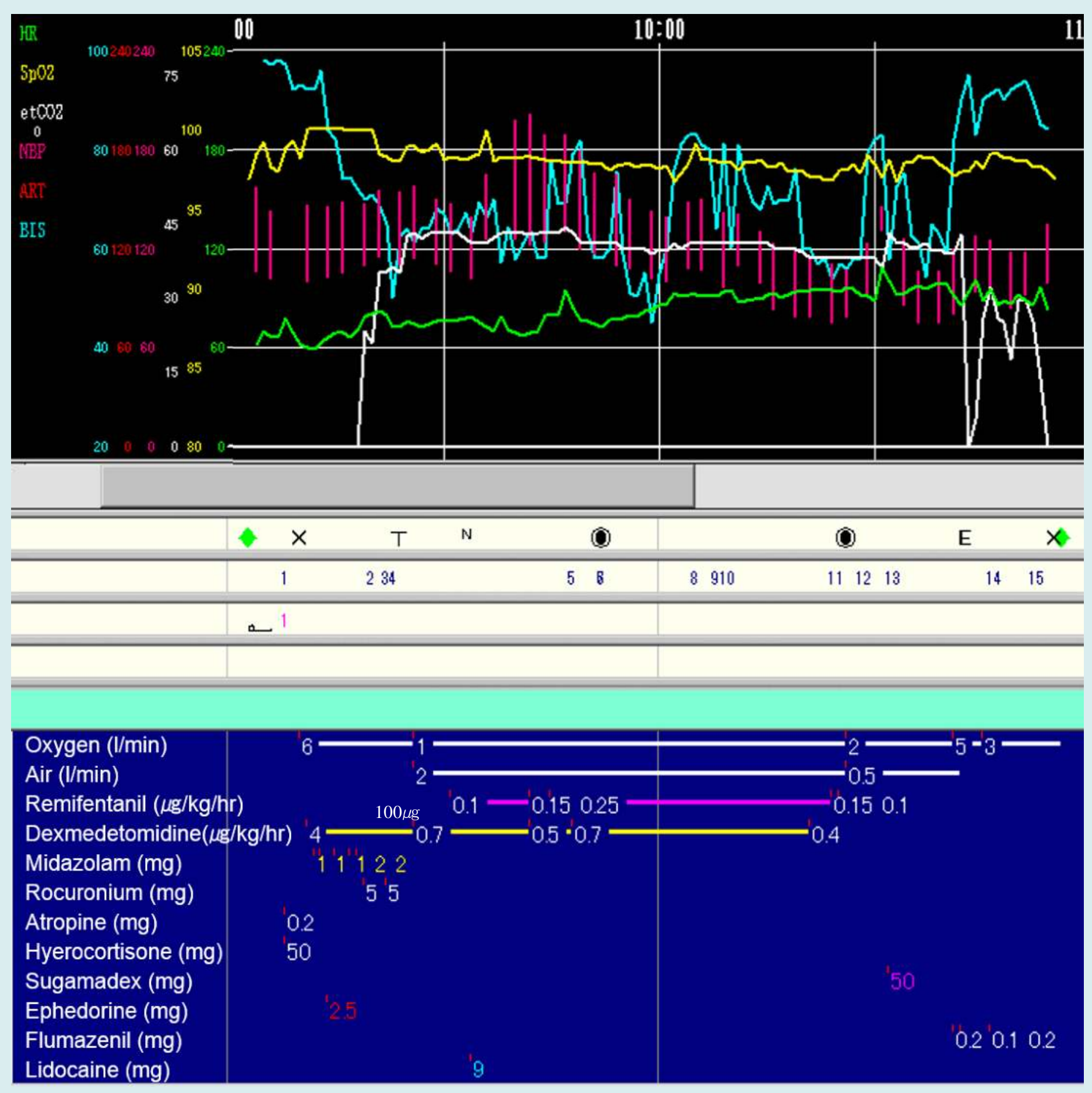

\section{Preparation}

- Wash out inhalational anesthetics (flushing oxygen at $10 \mathrm{~L} / \mathrm{min}$ for $30 \mathrm{~min}$ )

- Prepare intubation tubes of several sizes (\#5-7)

- Ready the airway management system, including a pediatric Airway scope ${ }^{\circledR}$,

a McGrath ${ }^{\circledR}$, Defibrillator, a Bispectal Index Score (BIS) monitor,

and a TOF monitor

Induction of anesthesia

- Load dexmedetomidine (DEX) $4 \mu \mathrm{g} / \mathrm{kg} / \mathrm{hr}$ for $15 \mathrm{~min}$

- Spray lidocaine around the larynx

- Add up to $7 \mathrm{mg}$ midazolam (MDZ)

- Administer $100 \mu \mathrm{g}$ remifentanil and $10 \mathrm{mg}$ rocuronium prior to intubation

- Intubate using a McGrath (tube size \#6)

Maintenance of anesthesia

- Use local anesthesia for the surgical field

- Continuously administer DEX and remifentanil

Extubation

- Administer sugammadex and flumazenil before extubation

- Continuously administer flumazenil after extubation

Postoperative management

- One-day (20 hr) stay in the ICU

- Titrate flumazenil according to the BIS score.

- Apply defibrillator pads until the next day.

- Check intraoperative or unpleasant memories during the postoperative visit

Our observations in this case

- Contrary to expectation, the susceptibility to treatment was normal.

- BIS score was high during the operation.

- The patient took longer than normal to regain a conscious state.

\section{Discussion}

- Despite the high BIS score, the patient had no intraoperative memory, suggesting the unreliability of the BIS score or existence of anterograde amnesia owing to MDZ.

- Drug used for sedation was excessive for smooth and clear awakening. Shorter-acting, non lipid-soluble agents might be better, but these are not routinely available.

\section{Learning points}

$\sim$ regarding anesthesia in patients with PTRF mutations

- Local anesthesia is recommended, if possible.

- Avoid using inhalation or lipid-soluble agents.

- Counterplan for long QT syndrome.

- Be aware of various complications of the disorder. 